\title{
Fundamental measure theory for non- spherical hard particles: predicting liquid crystal properties from the particle shape
}

\author{
René Wittmann ${ }^{1,2}$, Matthieu Marechal ${ }^{2}$ and Klaus Mecke ${ }^{2}$ \\ ${ }^{1}$ Department of Physics, University of Fribourg, Chemin du Musée 3, 1700 Fribourg, Switzerland \\ 2 Institut für Theoretische Physik, Universität Erlangen-Nürnberg, Staudtstr 7, 91058 Erlangen, Germany \\ E-mail: rene.wittmann@unifr.ch
}

\begin{abstract}
Density functional theory (DFT) for hard bodies provides a theoretical description of the effect of particle shape on inhomogeneous fluids. We present improvements of the DFT framework fundamental measure theory (FMT) for hard bodies and validate these improvements for hard spherocylinders. To keep the paper self-contained, we first discuss the recent advances in FMT for hard bodies that lead to the introduction of fundamental mixed measure theory (FMMT) in our previous paper (2015 Europhys. Lett. 109 26003). Subsequently, we provide an efficient semi-empirical alternative to FMMT and show that the phase diagram for spherocylinders is described with similar accuracy in both versions of the theory. Finally, we present a semiempirical modification of FMMT whose predictions for the phase diagram for spherocylinders are in excellent quantitative agreement with computer simulation results.
\end{abstract}

Keywords: non-spherical particles, colloids, liquid crystals, continuum theory

(Some figures may appear in colour only in the online journal)

\section{Introduction}

Hard-particle model systems help to improve our understanding of the effect of particle shape on collective behavior at a fundamental level. Furthermore, these system can now be realized in the form of colloids or nanoparticles due to recent advances in synthesis techniques [1]. A driving force for these efforts is the possibility that these experimental systems form structures that can be applied as novel materials. While these hard-particle systems were originally studied using computer simulations [2], the application of continuum theories is more natural for some long-wave-length or high-symmetry problems.

Density functional theory (DFT) $[3,4]$ is a continuum theory for systems that are inhomogeneous or anisotropic either due to applied external fields $[3,5,6]$ or spontaneous symmetry breaking [7-9]. Hard spheres represent a classical and quite tractable system to which density functional theory has been applied in many studies. One of the most successful versions of DFT for hard-sphere mixtures is Rosenfeld's fundamental measure theory (FMT), which is based on the fundamental measures of each spherical component, namely, its radius, area and volume [10]. A version of FMT derived from the zero-dimensional limit $[11,12]$ has proven to be very successful in predicting the properties of the crystal [8]. This FMT has been further modified to yield the excellent Carnahan-Starling equation of state [13] for the homogeneous fluid and the resulting FMT [14, 15] predicts the hard-sphere freezing transition very accurately [16].

Simultaneously, the interest in liquid crystals has been a motivation to apply DFT to anisotropic particles, for instance, hard spherocylinders, idealized rod-like molecules. The isotropic-smectic-A and nematic-smectic-A phase transitions of these rods were determined using different weighted density versions of DFT for anisotropic particles [17-20] and showed reasonable agreement with the essentially exact simulation results of [21]. However, the construction of the free energy functional of these theories is ad hoc and we would like to use a functional based solely on the geometry of the particles, such as FMT for hard spheres. Some fundamental 
measure functionals have been constructed for mixtures of hard spheres and specific anisotropic particles with zero volume, namely infinitely thin rods [22] and disks [23].

Rosenfeld extended his version of FMT to general anisotropic shapes [24]. However, this functional did not predict a stable nematic phase for any particle-shape due to the lack of additional measures which are sensitive to shape anisotropy. This deficiency was repaired by Hansen-Goos and Mecke $[5,6]$, who derived the so-called extended deconvolution FMT (edFMT) from the low-density limit by a truncated expansion and subsequent semi-empirical rescaling. The resulting isotropic-nematic transition $[6,25]$ shows excellent agreement with simulations [21]. In others studies of spherocylinders, the edFMT functional has been applied to systems under the influence of both static and time-dependent external fields that couple to the orientations of the particles $[25,26]$. The results for the time-dependent field, which were obtained using a dynamic version of density functional theory, were later confirmed to be qualitatively correct using computer simulations [27].

Equilibrium edFMT studies for a fluid (mixture) of polyhedra near a hard wall [28] confirmed the accuracy of the theory (with the sole exception of a pure inhomogeneous fluid of tetrahedra). A different study found the crystallization of rounded cubes with fixed orientations to be continuous for a surprisingly wide range of degrees of rounding [29], which was confirmed using simulations [29]. Whereas convexity is assumed at a rather fundamental level in the derivation of edFMT, the results for a fluid of dumbbells (fused pairs of hard spheres) near a hard wall [30] indicate that the theory is also quite accurate for mildly non-convex shapes.

The paper is organized as follows: first, we will discuss recent improvements to the theory [31, 32] that are necessary even for hard spherocylinders in section 2. The improved theory features mixed weighted densities, that is, weighted densities that are calculated by integrating over the degrees of freedom of more than one particle, whereas the other versions of FMT only contain one-body weighted densities. One can view edFMT as based on an expansion of the mixed weighted densities in terms of one-body tensorial weighted densities. To distinguish the improved theory from edFMT we have termed it fundamental mixed measure theory (FMMT) even though in most (but not all) cases the most efficient calculation of the mixed weighted densities is by expanding them in products of one-particle weighted densities as in edFMT; the difference between edFMT and such an implementation of FMMT is that the truncation order in the latter is increased until the expansion converges, while the maximum order is fixed in edFMT. While the inclusion of mixed weighted densities is the most profound change in the functional, a much less computationally involved modification [31] that preserves the maximum tensor order of edFMT already suffices to obtain a qualitatively correct phase diagram for short spherocylinders. This step is imperative to describe dense fluids of long rods, even in FMMT [32]. Still, of the FMT versions considered so far, only FMMT reproduces the exact low-density limit of the excess free energy for an inhomogeneous and/or anisotropic system (the low-density limit for the homogeneous isotropic phase of convex particles is exact for all FMT versions). As a result, the FMMT free energy is the only suitable FMT functional for problems such as the isotropic-nematic interfacial tension for very long spherocylinders where anisotropies and/or inhomogeneities persist down to low densities. We will obtain closed form expressions for the elastic constants of deformed nematic phases and the interfacial tension by mapping FMMT onto phenomenological theories.

In section 3, we will present further improvements of $\mathrm{F}(\mathrm{M}) \mathrm{MT}$ and new results obtained with these improved versions. In particular, we will present modifications to the second and the third term of the excess free energy functional of FMT. First, we will show that the rescaling of the second term [6] that depends on the (local) nematic order parameter has a similar accuracy as FMMT for most of the results we have obtained thus far for spherocylinders. Subsequently, we present a somewhat ad hoc rescaling of the third term in the excess free energy functional of FMMT and we show that this rescaling leads to an improved phase diagram for spherocylinders indicating the need for a more systematic modification of the third term.

Finally, we will summarize and discuss the improvements presented in this work and their generalizations to mixtures in section 4.

\section{Recent advances in fundamental measure theory}

DFT is based on the exact result that the density profile can be obtained by minimizing an expression of the form [3]

$$
\Omega[\rho]=\mathcal{F}_{\text {intr }}[\rho]+\sum_{i} \int \rho_{i}(\mathbf{r})\left(V_{\mathrm{ext}}^{(i)}(\mathbf{r})-\mu^{(i)}\right) \mathrm{d} \mathbf{r},
$$

with respect to the density profile $\rho_{i}(\mathbf{r})$, where $\mu^{(i)}$ is the chemical potential and $V_{\mathrm{ext}}^{(i)}$ is the external potential. In equation (1), we have used the notation of [24], in which $i$ denotes a specific shape, size and orientation for brevity (for the chemical potential, $\mu^{(i)}=\mu^{(j)}$ for all $j$ that denote rotated copies of $i$ ). Accordingly, the sum over $i$ denotes a sum over all species and, for each anisotropic species, an integral over its orientations. We will continue to use this notation throughout this paper. We will only consider one-component systems in this work, but write down the general framework of edFMT and FMMT for the more general case of mixtures of anisotropic particles. The functional $\mathcal{F}_{\text {intr }}[\rho]$ is independent of $V_{\text {ext }}^{(i)}(\mathbf{r})$, allowing reliable approximations based solely on the bulk phase behavior. In all FMTs, the intrinsic free energy is written as a sum,

$$
\mathcal{F}_{\text {intr }}[\rho]=\mathcal{F}_{\text {id }}[\rho]+\mathcal{F}_{\text {ex }}[\rho]=k_{\mathrm{B}} T \int\left(\Phi_{\text {id }}+\Phi_{\text {ex }}\right) \mathrm{d} \mathbf{r},
$$

where $k_{\mathrm{B}}$ is Boltzmann's constant, $T$ denotes the temperature, $\Phi_{\mathrm{id}}=\sum_{i} \rho_{i}\left(\ln \left(\rho_{i} \Lambda_{i}^{3}\right)-1\right)$ is the free energy density of the ideal gas (with $\Lambda_{i}$ the thermal wavelength) and

$$
\Phi_{\mathrm{ex}}=-n_{0} \log \left(1-n_{3}\right)+\frac{\phi_{2}}{1-n_{3}}+\frac{\phi_{3}}{\left(1-n_{3}\right)^{2}} .
$$


While the ideal gas part of the free energy density $\Phi_{\mathrm{id}}(\mathbf{r})$ is a function of the local one-body densities $\rho_{i}(\mathbf{r})$ at position $\mathbf{r}$, the excess free energy density $\Phi_{\mathrm{ex}}\left(\left\{n_{\nu}([\rho], \mathbf{r})\right\}\right)$, depends indirectly on the density profile through the local functionals $\phi_{2}([\rho], \mathbf{r})$ and $\phi_{3}([\rho], \mathbf{r})$ and the weighted densities

$$
n_{\nu}(\mathbf{r})=\sum_{i} \int \rho_{i}\left(\mathbf{r}_{1}\right) w_{i}^{(\nu)}\left(\mathbf{r}-\mathbf{r}_{1}\right) \mathrm{d} \mathbf{r}_{1}
$$

where $w_{i}^{(\nu)}(\mathbf{r})$ are weight functions of species/orientation $i$ and index $\nu \in\{0,1,2,3\}$; in general the $w^{(\nu)}$ contain $\delta$ distributions, see section 2.1 for their full definitions and further details.

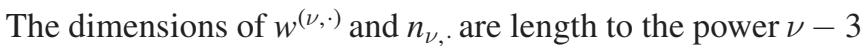
(in three spatial dimensions), where the $\cdot$ denotes possible other indices, which label, e.g. tensor components. The functional always has the form given by equation (3) in FMT (but not in some extensions [14, 15]); the differences between the various versions of FMT we will discuss lie in the definitions of $\phi_{2}$ and $\phi_{3}$. We have verified [31] that a modification along the lines of [15] yields only minor corrections to the presented results.

We will first describe the edFMT $[5,6]$ for general particle shapes and subsequently review recent improvements.

\subsection{Extended deconvolution fundamental measure theory}

In edFMT, the functional can be completely expressed in terms of weighted densities,

$$
\phi_{2}=n_{1} n_{2}-\vec{n}_{1} \cdot \vec{n}_{2}-\zeta \overleftrightarrow{n}_{1}: \overleftrightarrow{n}_{2},
$$

$\phi_{3}=\frac{3}{16 \pi}\left(\vec{n}_{2} \cdot \vec{n}_{2} \cdot \vec{n}_{2}-n_{2}\left|\vec{n}_{2}\right|^{2}+n_{2} \operatorname{Tr}\left[\overleftrightarrow{n}_{2}^{2}\right]-\operatorname{Tr}\left[\overleftrightarrow{n}_{2}^{3}\right]\right)$,

with $\operatorname{Tr}[A]$ equal to the trace of a matrix $A$ and $A: B \equiv \operatorname{Tr}[A B]$.

The set $\left\{w_{i}^{(\nu)}(\mathbf{r})\right\}$ is comprised of four scalar weight functions,

$$
\begin{gathered}
w_{i}^{(3)}(\mathbf{r})=\Theta\left(|\mathbf{r}|-R_{i}(\hat{\mathbf{r}})\right), \\
w_{i}^{(2)}(\mathbf{r})=\frac{1}{\mathbf{n}_{i}(\hat{\mathbf{r}}) \cdot \hat{\mathbf{r}}} \delta\left(|\mathbf{r}|-R_{i}(\hat{\mathbf{r}})\right), \\
w_{i}^{(1)}(\mathbf{r})=\frac{H_{i}(\hat{\mathbf{r}})}{4 \pi} w_{i}^{(2)}(\mathbf{r}), \\
w_{i}^{(0)}(\mathbf{r})=\frac{K_{i}(\hat{\mathbf{r}})}{4 \pi} w_{i}^{(2)}(\mathbf{r}),
\end{gathered}
$$

two vectorial weight functions,

$$
\begin{aligned}
\vec{w}_{i}^{(2)}(\mathbf{r}) & =\mathbf{n}_{i}(\hat{\mathbf{r}}) w_{i}^{(2)}(\mathbf{r}), \\
\vec{w}_{i}^{(1)}(\mathbf{r}) & =\mathbf{n}_{i}(\hat{\mathbf{r}}) w_{i}^{(1)}(\mathbf{r})
\end{aligned}
$$

and two tensorial weight functions of rank two,

$$
\begin{aligned}
\overleftrightarrow{w}_{i}^{(2)}(\mathbf{r}) & =\mathbf{n}_{i}(\hat{\mathbf{r}}) \mathbf{n}_{i}(\hat{\mathbf{r}})^{T} \\
\overleftrightarrow{w}_{i}^{(1)}(\mathbf{r}) & =\frac{\Delta \kappa_{i}(\hat{\mathbf{r}})}{4 \pi} \times\left[\mathbf{v}_{i}^{I}(\hat{\mathbf{r}}) \mathbf{v}_{i}^{I}(\hat{\mathbf{r}})^{T}-\mathbf{v}_{i}^{I I}(\hat{\mathbf{r}}) \mathbf{v}_{i}^{I I}(\hat{\mathbf{r}})^{T}\right] w_{i}^{(2)}(\mathbf{r}) .
\end{aligned}
$$

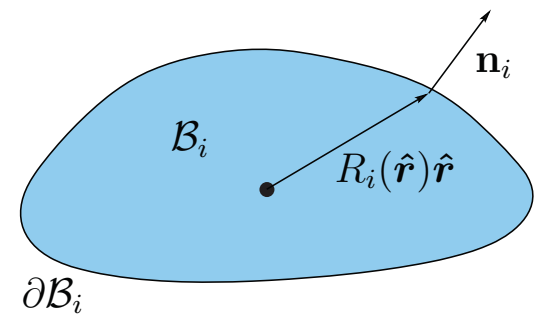

Figure 1. The surface $\partial \mathcal{B}_{i}$ of a body $\mathcal{B}_{i}$ is parametrized by a unit vector $\hat{\mathbf{r}}$, such that the corresponding point on the surface is $R_{i}(\hat{\mathbf{r}}) \hat{\mathbf{r}}$. The normal at this point is denoted by $\mathbf{n}_{i}(\hat{\mathbf{r}})$.

Here, the unit vector $\hat{\mathbf{r}}=\mathbf{r} /|\mathbf{r}|, R_{i}(\hat{\mathbf{r}})$ is the distance along $\hat{\mathbf{r}}$ from the origin, chosen to lie inside the particle $i$, to the surface and $\mathbf{n}_{i}(\hat{\mathbf{r}})$ is the normal to the surface at $R_{i}(\hat{\mathbf{r}}) \hat{\mathbf{r}}$, see figure 1(a). Moreover, $K_{i}(\hat{\mathbf{r}})=\kappa_{i}^{I}(\hat{\mathbf{r}}) \kappa_{i}^{I I}(\hat{\mathbf{r}})$ is the Gaussian curvature, $H_{i}(\hat{\mathbf{r}})=\frac{1}{2}\left(\kappa_{i}^{I}(\hat{\mathbf{r}})+\kappa_{i}^{I I}(\hat{\mathbf{r}})\right)$ is the mean curvature and $\Delta \kappa_{i}(\hat{\mathbf{r}})=\frac{1}{2}\left(\kappa_{i}^{I}(\hat{\mathbf{r}})-\kappa_{i}^{I I}(\hat{\mathbf{r}})\right)$ denotes the deviatoric curvature, where $\kappa_{i}^{I}(\hat{\mathbf{r}})$ and $\kappa_{i}^{I I}(\hat{\mathbf{r}})$ are the principal moments of the curvature in the directions $\mathbf{v}^{I}(\hat{\mathbf{r}})$ and $\mathbf{v}^{I I}(\hat{\mathbf{r}})$, respectively, at the point $R_{i}(\hat{\mathbf{r}}) \hat{\mathbf{r}}$ on the surface $\partial \mathcal{B}_{i}$ of the body $\mathcal{B}_{i}$, see figure 1 . Finally, $\Theta(\cdot)$ and $\delta(\cdot)$ denote the Heaviside and Dirac-delta functions, respectively.

Note that the presence of the denominator $\mathbf{n}_{i}(\hat{\mathbf{r}}) \cdot \hat{\mathbf{r}}$ of $w_{i}^{(2)}$ is caused by the specific parametrization $R_{i}(\hat{\mathbf{r}}) \hat{\mathbf{r}}$ that was chosen. This denominator is such that $\int \mathfrak{F}(\mathbf{r}) w_{i}^{(2)}(\mathbf{r}) \mathrm{d} \mathbf{r}=\int_{\partial \mathcal{B}_{i}} \mathfrak{F}(\mathbf{r}) \mathrm{d} A_{\mathbf{r}}$ for an arbitrary function $\mathfrak{F}$ where $\mathrm{d} A_{\mathbf{r}}$ is the area element of the surface $\partial \mathcal{B}_{i}$ at $\mathbf{r}$; in other words, including a factor of $w_{i}^{(2)}$ in an integral over the whole space has the sole effect of constraining the integral to the surface of the particle.

Hansen-Goos and Mecke [5, 6] derived edFMT starting from an expansion of the Mayer function $f_{i j}\left(\mathbf{r}_{1}-\mathbf{r}_{2}\right)$, which is -1 upon overlap of bodies $i$ and $j$ and zero otherwise, in terms of tensorial weight functions with increasing rank. The parameter $\zeta$ in equation (5) is a renormalization factor introduced by Hansen-Goos and Mecke [5, 6] to correct for the truncation of this expansion of the Mayer function after the term involving rank-two tensors. Common values for $\zeta$ are $\zeta=5 / 4$, which is obtained by minimizing the difference between the exact and edFMT excluded volumes [5] and $\zeta=1.6$, which results from a fit to simulation results for the isotropic-nematic (IN) transition [5]. The edFMT form of the functional as written here has the following drawbacks when considering liquid crystals of elongated particles: (i) The IN transition is not accurately described unless the fitted value $\zeta=1.6$ is used $[5,6]$. (ii) The width of the coexistence and the surface tension of the IN interface is too low [33]. (iii) The smectic-A phase is unstable for reasonable spherocylinder aspect ratios [31]. (iv) Finally, edFMT does not reduce to the Onsager functional in the limit of infinitely long particles, $L \rightarrow \infty$ and $\rho \rightarrow 0$, at fixed concentration $c \equiv L^{2} D \pi \rho / 4$, where $L$ and $D$ are the length and diameter, respectively, of the cylindrical section of the spherocylinder. As the Onsager functional is exact in that limit, this is a major drawback of edFMT. 
We will now consider a first modification of the original edFMT functional: the replacement of $\phi_{3}$ by a different expression [12] that leads to an improvement on points (ii) and (iii).

\subsection{Tarazona-Rosenfeld FMT}

Tarazona and Rosenfeld [12] completed the derivation (which was started in [11]) of FMT from a highly confining geometry, namely a cavity that is so small that it can hold only one particle. The free energy for such a system can be derived exactly and the derivation proceeds by demanding that the exact free energy is reproduced by the FMT functional at the highly peaked equilibrium density profile of this quasi-zero-dimensional system. The form of the free energy that was derived in this way differed from previous versions of FMT in the third term, $\phi_{3}$. Unfortunately, the form for $\phi_{3}$ from this derivation cannot be expanded in a finite number of weighted densities ${ }^{3}$. Therefore, an approximation was considered [12, 35], which leads to the following form for $\phi_{3}$,

$$
\phi_{3}=\phi_{3}^{(\mathrm{TR})}:=\frac{3}{16 \pi}\left(n_{2}^{3}-3 n_{2} \operatorname{Tr}\left[\vec{n}_{2}^{2}\right]+2 \operatorname{Tr}\left[\overleftrightarrow{n}_{2}^{3}\right]\right) .
$$

We will refer to edFMT with this version of $\phi_{3}$ theory as 'edFMT-TR'.

As can be seen in figure 2(a), this functional leads to a satisfactory agreement with the simulation results for spherocylinders with $L / D \lesssim 7$ when the semi-empirical parameter $\zeta$ is set to $5 / 4$ [31]. The improvement of edFMT-TR upon edFMT based on $\phi_{3}$ from equation (6) has been confirmed for the IN interfacial tension, the width and the location of the IN coexistence in the phase diagram and the equation of state (pressure as a function of packing fraction) for all isotropic and liquid crystal phases [31]. Most importantly, the smectic-A phase is stable in edFMT-TR for long enough spherocylinders as expected from simulation results [21] and the location of nematic-smectic-A (NSm-A) transition is predicted quite well. This is quite an impressive result as the free energy differences between different liquid crystal phases are usually small and, as a result, difficult to resolve (although edFMT-TR is not the first DFT to reproduce the topology of the spherocylinder phase diagram [17]). Unfortunately, the NSm-A transition, which is known to be first-order for all aspect ratios from simulations [21, 36], becomes secondorder above a certain aspect ratio, $7<L / D<8$, in edFMTTR. This spurious tricritical point is also seen in other DFTs [17, 37].

For longer spherocylinders, a strong increase of the nematic-smectic-A coexistence densities with increasing aspect ratio at fixed packing fraction is observed for the value of $\zeta=5 / 4$ and, in fact, any value of $\zeta$ in the allowed regime $0 \leqslant \zeta \leqslant 2$ that is not equal to $\zeta=2$. This increase of the NSm-A coexistence densities for $\zeta<2$ and the resulting eventual disappearance of the smectic-A phase for long rods is in contradiction with simulation results [21] and all earlier theoretical approaches [17, 18, 38]. Unfortunately, simply

\footnotetext{
${ }^{3}$ It is possible to write down a convergent expansion in terms of weight functions that contain spherical harmonics of the normal vector, which will be reported elsewhere [34].
}
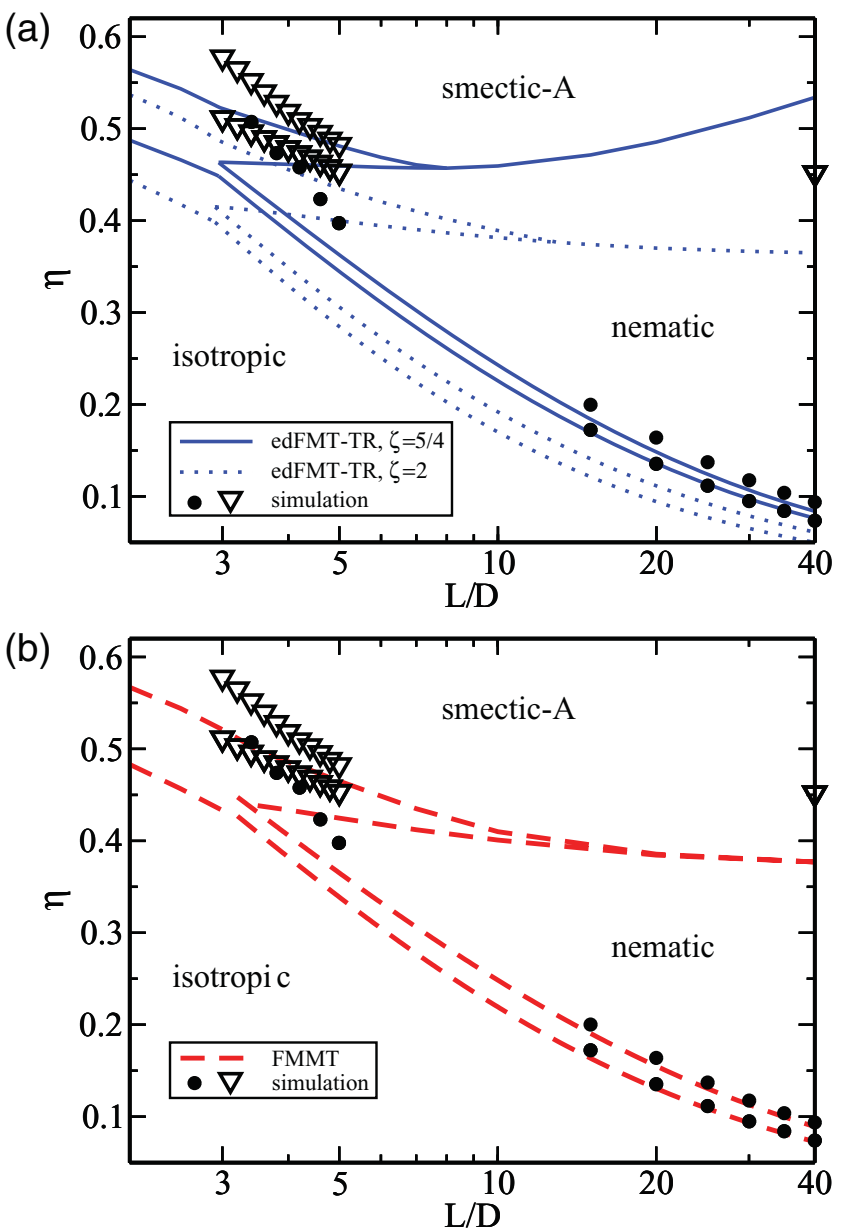

Figure 2. Phase diagram of hard spherocylinders in the $l-\eta$ representation, where $l=L / D$ is the aspect ratio, $\eta=\rho v=v N / V$ denotes the packing fraction, $N$ is the number of particles in the system, $V$ the volume of the system and $v$ is the volume of a particle. We compare our theoretical results obtained using edFMT with $\zeta=5 / 4$ and 2 [31] (a) and FMMT [32] (b) with simulation results by Bolhuis and Frenkel [21]. At small aspect ratios $l=L / D$, we observe a direct transition from the isotropic to the smectic-A phase. In all three theories, the nematic-smectic-A transition becomes (incorrectly) continuous beyond a certain aspect ratio. Panel (a) was reproduced from [31] (Copyright 2014, American Institute of Physics) and (b) from [32] (Copyright 2015, IOP Institute of Physics).

using $\zeta=2$ rather than $\zeta=5 / 4$ spoils the good agreement between edFMT-TR and simulations for $L / D \lesssim 7$ (below the tricritical point for $\zeta=5 / 4$ ). Clearly, a better approach than manually tweaking $\zeta$ to match simulation results is desirable.

In the next section, we will present such an approach, fundamental mixed measure theory. From now on, we will only use the Tarazona-Rosenfeld form of $\phi_{3}$, equation (14), although we should remark here that this version of $\phi_{3}$ does not lead to the excellent Percus-Yevick form for the direct correlation function $c_{i j}^{(2)}\left(\mathbf{r}=\mathbf{r}_{1}-\mathbf{r}_{2}\right):=-\beta \delta^{2} \mathcal{F}_{\mathrm{ex}} / \delta \rho_{i}\left(\mathbf{r}_{1}\right) \delta \rho_{j}\left(\mathbf{r}_{2}\right)$ of hard spheres, in contrast to $\phi_{3}$ from equation (6) [8]. Doing so, we achieve a further improvement on points (ii) and (iv) listed at the end of section 2.1, compared to edFMT-TR. 


\subsection{Fundamental mixed measure theory}

The exact expression for the low-density limit can be written in terms of an integral over a local functional,

$$
\lim _{\rho \rightarrow 0} \mathcal{F}_{\text {ex }}[\rho]=\int\left(n_{0} n_{3}+N_{12}\right) \mathrm{d} \mathbf{r}
$$

with the mixed weighted density $N_{12}$ and corresponding mixed weight function $\mathcal{W}^{(12)}$,

$$
\begin{gathered}
N_{12}(\mathbf{r}):=\sum_{i j} \iint \mathcal{W}_{i j}^{(12)}\left(\mathbf{r}-\mathbf{r}_{1}, \mathbf{r}-\mathbf{r}_{2}\right) \rho_{i}\left(\mathbf{r}_{1}\right) \rho_{j}\left(\mathbf{r}_{2}\right) \mathrm{d} \mathbf{r}_{1} \mathrm{~d} \mathbf{r}_{2}, \\
\mathcal{W}_{i j}^{(12)}:=\frac{\kappa_{i}^{I I}\left(\mathbf{v}_{i}^{I} \cdot \mathbf{n}_{j}\right)^{2}+\kappa_{i}^{I}\left(\mathbf{v}_{i}^{I I} \cdot \mathbf{n}_{j}\right)^{2}}{4 \pi\left(1+\mathbf{n}_{i} \cdot \mathbf{n}_{i}\right)} w_{i}^{(2)} w_{j}^{(2)},
\end{gathered}
$$

where in $\mathcal{W}_{i j}^{(12)}$ all quantities with species/orientation index $i$ or $j$ also implicitly depend on a spatial coordinate $\mathbf{r}-\mathbf{r}_{1}$ or $\mathbf{r}-\mathbf{r}_{2}$, respectively. These mixed weight functions are related to the mixed curvature measures from integral geometry [39], as we have detailed elsewhere [40]. The fact that these mixed curvature measures are used (albeit not calculated) regularly in integral geometry was the original motivation for trying to calculate $N_{12}$ directly, which we have done successfully for all liquid crystal phases of spherocylinders using a parametrized density profile [32].

The fundamental mixed measure functional can be obtained from the FMT functional, equation (3) by setting

$$
\phi_{2}=N_{12}
$$

From equation (15), we see that this choice leads to an exact functional in the low density limit. The phase diagram for spherocylinders as predicted by FMMT is shown in figure 2(b), where as in edFMT-TR we used the Tarazona-Rosenfeld form, equation (14), for $\phi_{3}$. The agreement for $L / D \lesssim 7$ is still good, although edFMT-TR with $\zeta=5 / 4$ is slightly more accurate than FMMT for the NSm-A transition due to a cancellation of errors in that case (as the $\zeta$ parameter was introduced to correct for truncation errors, any deviation of edFMT-TR results from the non-truncated FMMT should be considered an error). The spurious increase of the NSm-A transition with increasing rod length in edFMT-TR with $\zeta<2$ is not found in FMMT as we anticipated in section 2.2. Most importantly, the results for long rods at low density have the kind of accuracy we would expect from a DFT; in particular, the width of the IN transition is reproduced by FMMT quite nicely. Similar improvement has been found also for the IN interfacial tension (not shown) [32], which is strongly correlated with the width of the IN coexistence.

For the smectic-A phase and the isotropic-nematic interface, we calculated the free energy for a parametrized density profile [32] using Monte Carlo integration ${ }^{4}$ with recursive stratified sampling [41, 42]. These calculations are very computationally intensive, which is why we proposed an

\footnotetext{
${ }^{4}$ We have performed the integrals in the mixed weighted density directly in real space; we did not consider the possible optimization of using Fourier transforms to perform the convolution-like integrals.
}

expansion of $N_{12}$ in tensors [32] that converges more quickly than the tensor expansion that was originally proposed [6]. Alternatively, one can use Wertheim's expansion of the Mayer bond in spherical harmonics [43], which we will report on elsewhere [34]. We recommend that such an expansion is used whenever it converges quickly, which is guaranteed if the expansion of the orientational distribution in spherical harmonics converges quickly. We repeat that one should use a high enough order to ensure that the expansion has converged to obtain FMMT.

\subsection{Mapping onto phenomenological theories}

The complexity of the problems for which DFT is designed and the microscopic nature of FMT lead to a necessarily complex formulation of the theory; therefore, analytical results are rare. Phenomenological approaches, such as Landau-de Gennes [44, 45] theory, frequently lead to analytical formulas in terms of their adjustable constants, which usually have no direct microscopic meaning. These prefactors can be fixed by comparing with the predictions from a microscopic DFT for some simple problems. We have used such a procedure in two (related) cases; further work is required to generalize the procedure to other cases, especially deformations of smectic-A phases or crystals.

In the following, we only consider one-component systems of uni-axial rods, so we will write $\rho(\mathbf{r}, \hat{u})$, instead of $\rho_{i}(\mathbf{r})$, for the density profile of a particle with orientation $\hat{\mathbf{u}}$ and position $\mathbf{r}$, where the orientation is given by the direction $\hat{\mathbf{u}}$ of the particle's long axis.

First, we consider the Frank elastic energy for long-wavelength deformations of the director field $\hat{n}(\mathbf{r})$ (local axis of preferred alignment) in the one-component nematic phase,

$$
\begin{aligned}
\mathcal{F}_{\mathrm{d}}[\hat{\mathbf{n}}]= & \frac{1}{2} \int \mathrm{d} \mathbf{r}\left(K_{1}(\nabla \cdot \hat{\mathbf{n}})^{2}+K_{2}(\hat{\mathbf{n}} \cdot(\nabla \times \hat{\mathbf{n}}))^{2}\right. \\
& \left.+K_{3}(\hat{\mathbf{n}} \times(\nabla \times \hat{\mathbf{n}}))^{2}\right),
\end{aligned}
$$

where the prefactors $K_{\epsilon}$, with $\epsilon \in\{1,2,3\}$, are called the Frank elastic constants and the three terms penalize different modes of deformation, called splay, twist and bend.

By considering the free energy of a deformed nematic liquid crystal explicitly from a microscopic point of view and comparing to equation (19), Poniewierski and Stecki were able to express the Frank elastic constants in terms of the direct correlation function $c^{(2)}\left(\mathbf{r}, \hat{\mathbf{u}}_{i}, \hat{\mathbf{u}}_{j}\right) \equiv c_{i j}^{(2)}(\mathbf{r})$ in the undeformed nematic phase. In this case, the expression for $c^{(2)}\left(\mathbf{r}, \hat{\mathbf{u}}_{1}, \hat{\mathbf{u}}_{2}\right)$ from edFMT(-TR) or expanded FMMT (up to a finite order) is simple enough that we were able to find analytic (albeit lengthy) expressions for $K_{\epsilon}$ using Piewierski and Stecki's method [40]. As an example, we give the expansion of the elastic constants to quadratic order in the order parameter

$$
S=\int \mathrm{d} \hat{\mathbf{u}}\left(\frac{3}{2}\left[\hat{\mathbf{u}} \cdot \hat{\mathbf{n}}_{0}\right]^{2}-\frac{1}{2}\right) \frac{\rho(\hat{\mathbf{u}})}{\rho}
$$

of the homogeneous and unperturbed nematic phase with density profile $\rho(\mathbf{r}, \hat{\mathbf{u}})=\rho(\hat{\mathbf{u}})$, where $\rho=\int \mathrm{d} \hat{\mathbf{u}} \rho(\hat{\mathbf{u}})=N / V$ is the 

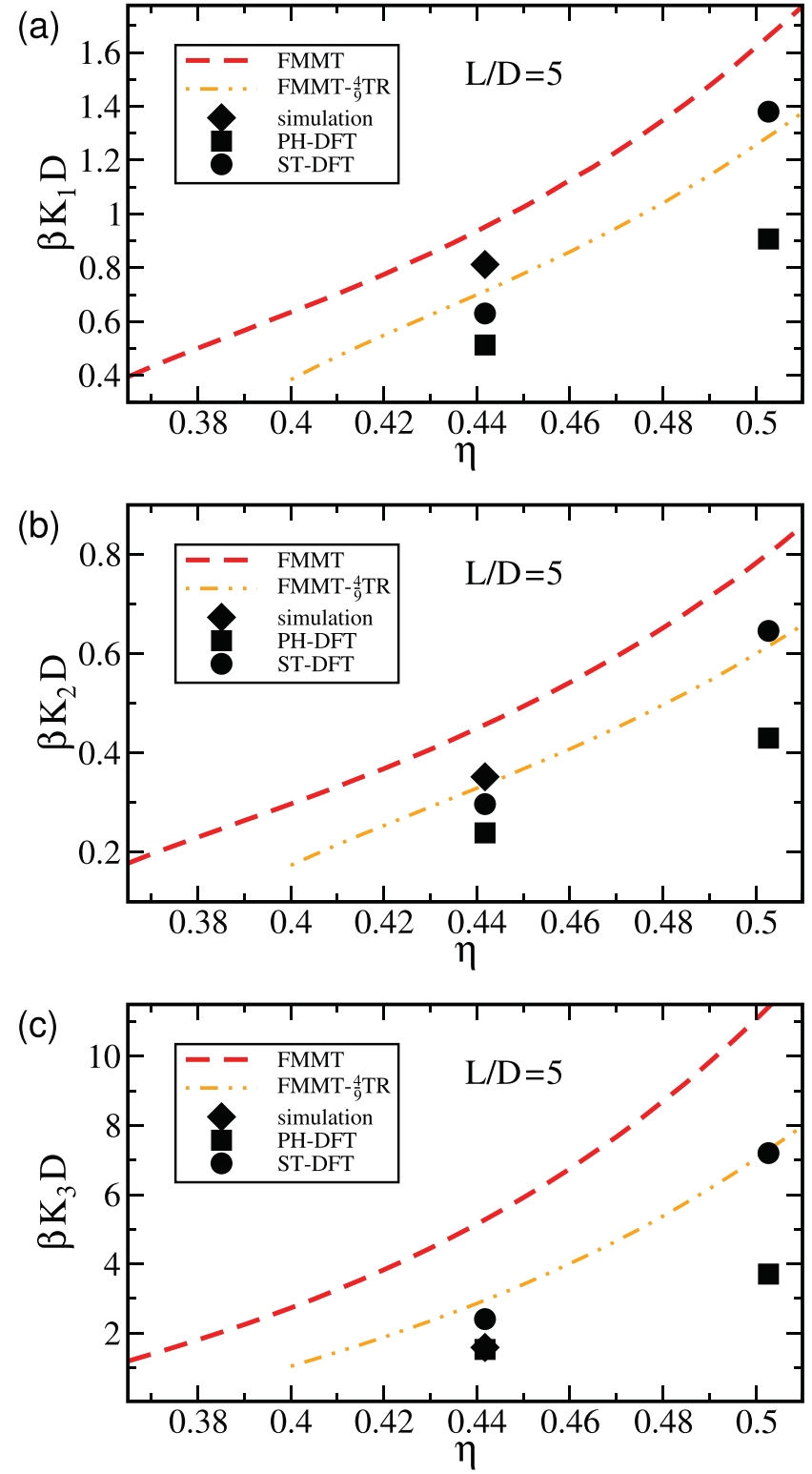

Figure 3. The elastic constants, $K_{1}, K_{2}$ and $K_{3}$ corresponding to splay, twist and bend deformation, respectively, for hard spherocylinders: a comparison between FMMT results reproduced from [40] (dashed lines), FMMT- $\frac{4}{9}$ TR results from this work (dotdashed lines), predictions from other DFTs (PH-DFT [49]: squares; ST-DFT [48]: circles) and simulation results [47] (diamonds). FMMT $-\frac{4}{9}$ TR was obtained from FMMT by a semi-empirical rescaling of $\phi_{3}$, see section 3.2.

orientationally averaged bulk density and $\hat{\mathbf{n}}_{0}$ is the undistorted nematic director.

The tensor expansion of FMMT leads to expressions that can be resummed to the closed formulas

$$
\begin{aligned}
\beta K_{1}^{(\mathrm{MM})} D= & \frac{135 \eta^{2} l^{2}\left(l^{2}+2\right)}{28 \pi(1-\eta)(3 l+2)^{2}} S^{2} \\
& +\frac{81 \eta^{3} l^{2}(l+1)\left(l^{2}+1\right)}{7 \pi(1-\eta)^{2}(3 l+2)^{3}} S^{2}+\mathcal{O}\left(S^{3}\right),
\end{aligned}
$$

$$
\begin{aligned}
\beta K_{2}^{(\mathrm{MM})} D= & \frac{45 \eta^{2} l^{2}\left(l^{2}+9\right)}{28 \pi(1-\eta)(3 l+2)^{2}} S^{2} \\
& +\frac{27 \eta^{3} l^{2}(l+1)\left(2 l^{2}+9\right)}{14 \pi(1-\eta)^{2}(3 l+2)^{3}} S^{2}+\mathcal{O}\left(S^{3}\right)
\end{aligned}
$$

and $K_{3}^{(\mathrm{MM})}=K_{1}^{(\mathrm{MM})}+\mathcal{O}\left(S^{3}\right)$ [40], which, in addition to the nematic order parameter $S$, only depend on the rod aspect ratio $l=L / D$ and the packing fraction $\eta=\rho v$, where $v=\pi L D^{2} / 4+\pi D^{3} / 6$ is the volume of a spherocylinder. With these expressions or the full expansions from [40], the Frank elastic theory becomes a microscopic theory for long-wavelength deformations of the nematic phase, which requires as input only these three parameters. As a result, all applications of the Oseen-Frank theory [46] can be directly translated to systems of hard spherocylinders with no adjustable constants other than $S$.

The numerical Frank constants from FMMT [40] are compared to simulation results [47] and the predictions from more ad-hoc DFTs [48, 49] in figure 3. Note that the difficulty of obtaining these elastic constants in computer simulation leads to a scarcity of available results: there is only one simulation data point (the diamond) for each of the elastic constants in figure 3. The results from FMMT, especially for $K_{3}$ are somewhat less accurate than the results from other DFTs. We propose a semi-empirical modification of the FMMT functional in section 3.2 that leads to improved results for the elastic constants (among others).

A second empirical theory is Landau-de Gennes theory $[44,45]$ where the free energy is again written as an integral over a local free-energy density

$$
\Omega[Q]=\beta^{-1} \int_{V} \mathrm{~d} \mathbf{r}\left[\omega_{\mathrm{b}}\left(Q_{\alpha \beta}(\mathbf{r})\right)+f_{\mathrm{d}}\left(Q_{\alpha \beta, \gamma}(\mathbf{r})\right)\right]
$$

(the density of the grand potential our case) that depends on the order tensor $Q_{\alpha \beta}(\mathbf{r})=\int \mathrm{d} \hat{\mathbf{u}}\left(\frac{3}{2} \hat{\mathbf{u}}_{\alpha} \hat{\mathbf{u}}_{\beta}-\frac{1}{2} \delta_{\alpha \beta}\right) \rho(\mathbf{r}, \hat{\mathbf{u}}) / \rho(\mathbf{r})$, where $\rho(\mathbf{r})=\int \mathrm{d} \hat{\mathbf{u}} \rho(\mathbf{r}, \hat{\mathbf{u}})$, and its derivatives $Q_{\alpha \beta, \gamma}:=\partial Q_{\alpha \beta} / \partial r_{\gamma}$. The local free energy can be written as the sum of a bulk contribution $\omega_{\mathrm{b}}$, which is the free energy of a (unaxial) nematic phase to fourth order in the components $Q_{\alpha \beta}$ of the order tensor, and the free energy of deformation, which is equivalent to the Frank elastic energy to second order in the derivatives $Q_{\alpha \beta, \gamma}$ (assuming low orientational order, the Frank elastic energy can be obtained from the Landau-de Gennes free energy of deformation by setting $Q_{\alpha \beta}(\mathbf{r})=S\left(\hat{\mathbf{n}}_{\alpha}(\mathbf{r}) \hat{\mathbf{n}}_{\beta}(\mathbf{r})-\frac{1}{3}\right)$ with $S$ constant).

For the IN interface, the free energy can be written as a square gradient form containing only a local scalar order parameter [45], so that [50] we can analytically calculate the density profile and the interfacial tension $\gamma \equiv\left(\Omega-\Omega_{\mathrm{b}}\right) / A$ where $\Omega_{\mathrm{b}}$ is the bulk grand potential at the coexistence chemical potential and $A$ the interfacial area. Using the analytical formulas for the Frank elastic constants, equations (21) and (22), and fixing the remaining prefactors in the phenomenological free energy by comparing to bulk properties [33, 40], we obtain the analytical formula for the dimensionless interfacial tension 
(a)

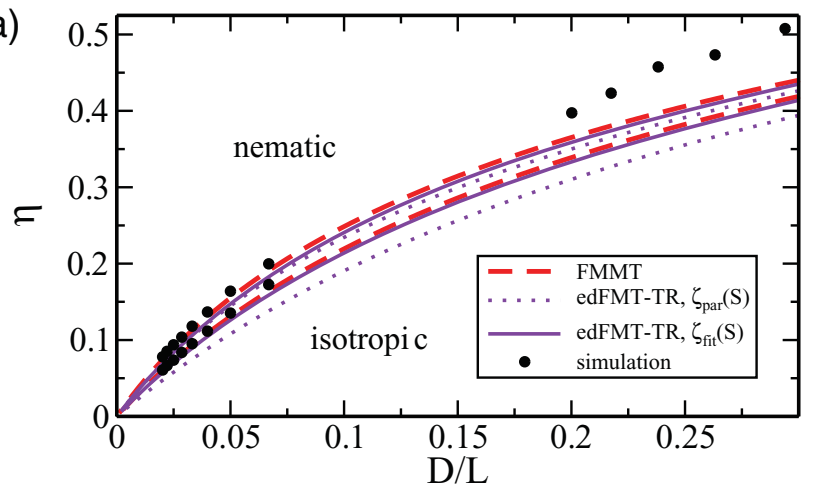

(b)

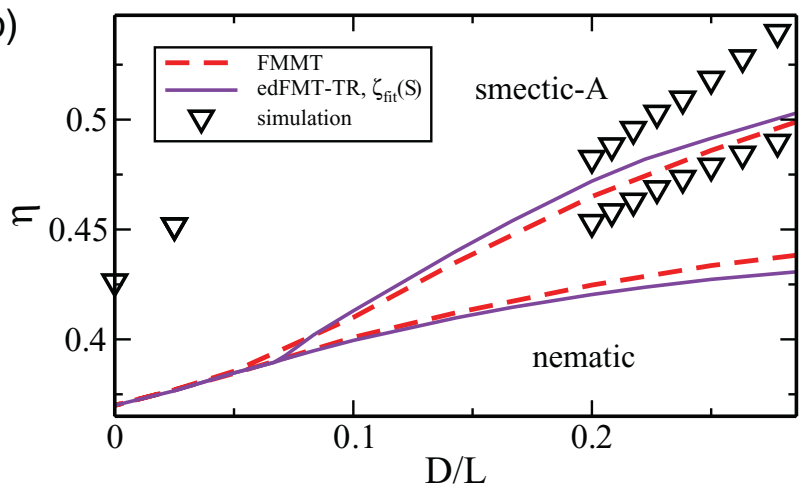

Figure 4. The coexistence of (a) the isotropic and the nematic and (b) the nematic and the smectic-A phases from FMMT and edFMTTR with the $\zeta_{\text {fit }}(S)$ approximation in equation (25): coexistence packing fractions as a function of the inverse aspect ratio $D / L$. In the first case, we also include the results with the parabolic approximation $\zeta_{\text {par }}(S)$ from [6].

$$
\beta \gamma_{\mathrm{t}}^{(\mathrm{MM})} D^{2}=b_{\mathrm{t}}\left(l, \eta_{\mathrm{N}}\right) \sqrt{\Delta \omega_{\mathrm{m}} v} S_{\mathrm{N}},
$$

where $\eta_{\mathrm{N}}$ and $S_{\mathrm{N}}$ are the packing fraction and nematic order parameter, respectively, of the nematic phase at IN coexistence. Furthermore, $\Delta \omega_{\mathrm{m}} v=\max _{0<S<S_{\mathrm{N}}}\left[\omega_{\mathrm{b}}\left(S, \mu_{\mathrm{c}}\right)-\omega_{0}\left(\mu_{\mathrm{c}}\right)\right] v$ is the maximum dimensionless grand-potential density for a non-equilibrium homogeneous nematic phase with order parameter $0<S<S_{\mathrm{N}}$ at the coexistence chemical potential $\mu=\mu_{\mathrm{c}}$ relative to the value $\omega_{0}\left(\mu_{\mathrm{c}}\right)$ for the isotropic phase at coexistence. Finally, the prefactor in equation (24) reads $b_{\mathrm{t}}\left(l, \eta_{N}\right)=l \eta_{\mathrm{N}}\left[15 l^{3}\left(5-\eta_{\mathrm{N}}\right)+10 l^{2}\left(5+\eta_{\mathrm{N}}\right)+72 l\left(5-3 \eta_{\mathrm{N}}\right)+\right.$ $\left.48\left(5-2 \eta_{\mathrm{N}}\right)\right]^{1 / 2} \sqrt{8 / 21} /\left[\pi\left(1-\eta_{\mathrm{N}}\right)(3 l+2)^{2}\right]$ for the equilibrium alignment of the director, which is tangential to the interface. The result, equation (24), for the dimensionless surface tension has the form of a geometric average of typical (nondimensionalized) values, $\left[b_{\mathrm{t}}\left(l, \eta_{N}\right) S_{N}\right]^{2}$ and $\Delta \omega_{m} v$, for the deformation free energy $f_{\mathrm{d}}$ and the bulk free energy difference $\omega_{\mathrm{b}}-\omega_{0}$, respectively (see also [33, 45], which have slightly different definitions of $b_{\mathrm{t}}$ ).

This expression [51] performs quite well for the IN interfacial tension when compared with direct results from FMMT (not shown), where $S_{\mathrm{N}}, \eta_{\mathrm{N}}$ and $\Delta \omega_{\mathrm{m}}$, which only depend on the aspect ratio, are calculated using FMMT.

\section{Further improvements of FMT}

In this section, we discuss two possibilities to adapt FMT for anisotropic shapes which are of a somewhat empirical nature. The presented results show that, first of all, it is not always necessary to employ the full mixed weighted density or a high-order expansion and, secondly, that FMMT with $\phi_{3}$ from equation (14) can be further improved upon. In particular, the predictions for the IN transition at intermediate aspect ratios (point (i) at the end of section 2.1) can be improved.

\subsection{Order parameter dependent $\zeta$ correction}

While the computational complexity of FMMT is required to accurately describe very long rods at low packing fractions, edFMT-TR with an appropriate choice for $\zeta$ already exhibits a similar accuracy as FMMT for intermediate packing fractions. Unfortunately, the optimal choice for $\zeta$ not only depends on the specific shape of the particle; it also depends on the density profile. Hansen-Goos and Mecke [6] assumed that the optimal choice of $\zeta$ depends only on the degree of alignment, measured using the nematic order parameter $S$, for hard spherocylinders (for other families of shapes, it would also depend on the aspect ratio). The proposed $S$-dependent $\zeta$ correction, $\zeta(S)$, was determined by numerically minimizing the mean square deviation of the FMT excluded volume from the exact result with respect to $\zeta$ in the nematic phase with order parameter $S$ [6]. (The excluded volume is defined as $\int \mathrm{d} \mathbf{r} f\left(\mathbf{r}, \hat{\mathbf{u}}_{1}, \hat{\mathbf{u}}_{2}\right)$, where $f\left(\mathbf{r}, \hat{\mathbf{u}}_{1}, \hat{\mathbf{u}}_{2}\right)$ is the Mayer function. $)$

It is convenient for the minimization of FMT to have an analytical approximation for $\zeta(S)$. We propose the following form

$$
\zeta_{\mathrm{fit}}(S)=\sum_{k=0}^{k_{\mathrm{t}}} a_{k} S^{k}+b_{1} \sqrt{1-S}+b_{2}\left(S+\frac{1}{2}\right)^{q},
$$

where the $\sqrt{1-S}$ dependence of the second term is essential to obtain the correct scaling of the order parameter $S=1+\mathcal{O}\left(l^{-2}\right)$ with $l \equiv L / D$ at finite packing fraction [31]. This term is missing from the earlier parabolic approximation $\zeta_{\text {par }}(S)$ of Hansen-Goos and Mecke [6]. We further included a term with an exponent $1<q<2$ that allows the second derivative of $\zeta_{\text {fit }}(S)$ to similarly diverge at the other extremal value $S=-1 / 2$; this seems to be a possibility if we consider the numerical data [6]. In the following, we use equation (25) including the polynomial in $S$ of degree $k_{\mathrm{t}}=5$, impose the conditions $\zeta(0)=5 / 4, \zeta(1)=2, \zeta(-1 / 2)=16 / 11$ and $\zeta^{\prime}(0)=0$ and determine the remaining parameters through a fit to $\zeta(S)$. We obtain $a_{0}=-27.90, a_{1}=-65.87, a_{2}=-1.229$, $a_{3}=0.7880, \quad a_{4}=-1.233, \quad a_{5}=0.3174, \quad b_{1}=-2.525$, $b_{2}=64.23$ and $q=1.02^{5}$.

With this fitted $\zeta(S)$ we obtained the IN interfacial tension and liquid crystal phase diagram for spherocylinders. For all bulk phases, $S$ is constant as we used an approximation for the

\footnotetext{
${ }^{5}$ Note that the fitted value for $q$ is close to one and, thus, $b_{2}$ and $a_{1}$ are strongly correlated. However, we were unable to obtain a satisfactory fit for $S<0$ without the $\left(S+\frac{1}{2}\right)^{q}$ term.
} 


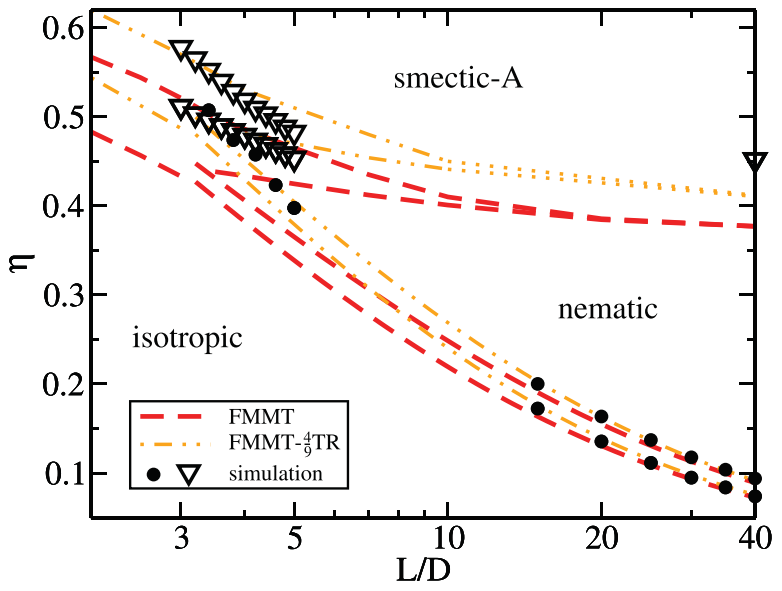

Figure 5. The phase diagram for spherocylinders, packing fraction $\eta$ versus the aspect ratio $L / D$. We compare results from FMMT with $\phi_{3}$ from equation (14) and FMMT- $\frac{4}{9} \mathrm{TR}$, for which $\phi_{3}$ is given by equation (27), with simulation results [21]. The dotted lines in the second case indicate the extrapolation to a continuous transition at $\eta=0.4056$ in the limit $L / D \rightarrow \infty$.

density profile of the smectic-A phase where the orientational order is independent of the position [31]. For the IN interface, we used [6] $S(\mathbf{r})=S(z)=\int \operatorname{d} \hat{\mathbf{u}}\left(\frac{3}{2}\left(\hat{\mathbf{u}} \cdot \hat{\mathbf{n}}_{0}\right)^{2}-\frac{1}{2}\right) \rho(\mathbf{r}, \hat{\mathbf{u}}) / \rho(\mathbf{r})$. Generally, we find very good agreement between FMMT and the $\zeta_{\text {fit }}(S)$ approximation [51] (not all results are shown); as an example, we show the IN and the NSm-A transition as a function of inverse aspect ratio $D / L$ in figure 4 . Both phase transitions are quite well described by the theory with the $\zeta_{\text {fit }}(S)$ correction, but the IN transition is poorly described by $\zeta_{\text {par }}(S)$ from $[6]^{6}$. Reassuringly, the predictions of edFMTTR with $\zeta_{\text {fit }}(S)$ and FMMT for the density of the (incorrectly continuous) NSm-A transition coincide exactly for infinitely long rods, whereas $\zeta_{\text {par }}(S)$ yields the same results as a constant $\zeta=2$, see figure $2(\mathrm{a})$.

We should note that the approach of [6] is not the only possibility for rescaling the $\overleftrightarrow{n}_{1}: \overleftrightarrow{n}_{2}$ term in $\phi_{2}$. One could also have approximated $\phi_{2}(\mathbf{r})$ as

$$
\phi_{2} \simeq n_{1} n_{2}-\vec{n}_{1} \cdot \vec{n}_{2}-\overleftrightarrow{n}_{1}: \overleftrightarrow{n}_{2} \zeta\left(\frac{\overleftrightarrow{n}_{1}: \overleftrightarrow{n}_{2}}{n_{1} n_{2}}\right),
$$

where the parameter $\zeta$ in equation (5) is replaced by a function $\zeta(x)$ of $x=\overleftrightarrow{n}_{1}: \overleftrightarrow{n}_{2} /\left(n_{1} n_{2}\right)$ instead of a function $\zeta(S)$ of $S$. Then one could determine $\zeta(x)$ in the nematic phase described by the parametrized density profile of [6] (by fitting $\zeta(x)$ to numerical data for $n_{12} / \overleftrightarrow{n}_{1}: \overleftrightarrow{n}_{2}$, where $n_{12}:=n_{1} n_{2}-\vec{n}_{1} \cdot \vec{n}_{2}-N_{12}$ ).

This approach has the advantage that the rescaling is formulated solely in terms of weighted densities. As such, it is directly applicable to inhomogeneous systems and there is no ambiguity on how to define $S(\mathbf{r})$ locally as for the $\zeta(S)$ approach. For the liquid crystal phase diagram we have presented here, we do not expect a large improvement as the values for $\zeta$ are likely very similar in both approaches in the

\footnotetext{
${ }^{6}$ From the predictions of edFMT with equation (6) for $\phi_{3}$, the opposite conclusion can be drawn, i.e. the better fit to the excluded volume shows the worst agreement with simulation results [6].
}

relevant case, i.e. whenever $\overleftrightarrow{n}_{1}: \overleftrightarrow{n}_{2}$ is large. However, it would be interesting to consider the $\zeta\left(\overleftrightarrow{n}_{1}: \overleftrightarrow{n}_{2} /\left(n_{1} n_{2}\right)\right)$ approach for strongly inhomogeneous anisotropic fluids, such as a nematic phase near a hard wall.

\subsection{Rescaling of $\phi_{3}$}

The prefactor $3 /(16 \pi)$ in both forms for $\phi_{3}$, equations (6) and (14), is obtained by rescaling to match the third virial coefficient for the homogeneous and isotropic fluid of spheres. There is no guarantee that this particular prefactor is the best for other shapes.

In a recent publication [31], we determined a suitable value for the prefactor by considering a simpler problem (than the full phase diagram for hard spherocylinders): the homogeneous phase of perfectly aligned hard particles. We showed that our FMMT equation of state (EOS) for aligned cylinders reduces to the results from an FMT specifically derived for parallel cylinders [52] if we set

$$
\phi_{3}=\frac{4}{9} \phi_{3}^{(\mathrm{TR})} .
$$

This choice, which we will refer to as 'FMMT- $\frac{4}{9} \mathrm{TR}$ ', has the advantage that the prefactor applies for all aspect ratios of cylinder-like particles, as the phase behavior of the hard parallel cylinder system is independent of the cylinder aspect ratio [52]. We showed that FMMT- $\frac{4}{9} \mathrm{TR}$ accurately predicts the properties of the IN transition of infinitely thin disks [31], which is arguably the simplest phase transition where the third virial coefficient matters [53]. In fact, our modified FMMT is about as accurate for the IN transition as an FMT-like DFT developed specifically for hard disks [23], see [31].

Now we consider the question of whether a rescaling of this kind could result in a better functional for spherocylinders by examining the liquid crystal phase behavior obtained with $\phi_{3}$ from equation (27). We show the phase diagram from FMMT $-\frac{4}{9}$ TR together with the results from the FMMT without rescaling and simulation data in figure 5. Clearly, including the prefactor 4/9 yields a much better agreement with the simulation results of Bolhuis and Frenkel [21] than for the 'bare' FMMT, in particular, for the NSm-A transition. In addition, the Frank elastic constants are predicted with higher accuracy by FMMT- $\frac{4}{9}$ TR than by FMMT, especially for $K_{3}$, as shown in figure 3. This improvement for the $K_{\epsilon}$ is mostly caused by more accurate predictions for the nematic order parameter, on which the elastic constants depend strongly.

Most quantities at intermediate packing fractions are predicted more accurately by FMMT- $\frac{4}{9}$ TR than by FMMT [51], while the results at low packing fraction remain accurate as we have only rescaled the $\phi_{3}$ term, which does not affect the leading-order term, equation (15), in the density expansion. A counterexample is the EOS of the nematic and smectic-A phases for spherocylinders with $L / D=5$ [51], which becomes (slightly) less accurate by the rescaling. In addition, the third order virial coefficient (of the isotropic phase) [54] is underestimated already by FMT and rescaling $\phi_{3}^{(\mathrm{TR})}$ by any factor smaller than one makes the disagreement worse, as it decreases the 
Table 1. The consecutive improvements made to Rosenfeld's fundamental measure theory [10] (more precisely, Tarazona's version [8]) to obtain a suitable density functional theory for non-spherical particles.

\begin{tabular}{lll}
\hline Designation and reference & Modification & Main advantage \\
\hline edFMT [5, 6] & Rank-two tensors added to $\phi_{2}$, see equation (5) & Stable nematic phase \\
edFMT-TR [31] & $\phi_{3}$, equation (6), replaced by $\phi_{3}^{\text {TR }}$, equation (14) & Stable smectic phase \\
FMMT [32] & Implemented exact $\phi_{2}=N_{12}$, see equation (18) & Exact low-density limit \\
edFMT-TR, $\zeta(S)$ [6] (this work) & Reverted to $\phi_{2}$ from equation (5) but with $\zeta=\zeta(S)$ & Efficient compared to FMMT \\
FMMT- $-\frac{4}{9}$ TR [31] (this work) & $\phi_{2}=N_{12}$ and rescaled $\phi_{3}^{\text {TR }}$, see equation (27) & Improved phase diagram \\
\hline
\end{tabular}

Notes: Each modification is applied in addition to the modifications listed before it. The $\zeta(S)$ modification, where the semi-empirical prefactor $\zeta$ depends on the nematic order parameter $S$ defined in equation (20), was proposed in [6] for edFMT (with $\phi_{3}$ from [12]); in this work, we implemented this modification in edFMT-TR (with $\phi_{3}^{\mathrm{TR}}$ from [12]). The improvements made in [14, 15, 55, 56] to improve the FMT prediction for the hard-sphere equation of state are not contained in this table.

third virial coefficient from FMT (the isotropic EOS is the same for FMMT and FMT). However, increasing the factor in front of $\phi_{3}$ to obtain a more accurate third virial coefficient would ruin the reasonable accuracy of the FMT equation of state for the isotropic phase (away from the low-density limit), which is mostly accurate due to a cancellation of errors: at low densities the pressure increases too slowly because FMT underestimates the low-order virial coefficients, while the increase of the pressure at higher densities is overestimated by FMT (presumably because FMT underestimates the short-ranged order that leads to improved local packing in the fluid).

Finally, the value 4/9 is a bit arbitrary; in fact, the EOS of infinitely long parallel spherocylinders from FMMT- $\frac{4}{9} \mathrm{TR}$ does not reduce to the EOS from the FMT for parallel hard cylinders. As an infinitely long spherocylinder and an infinitely long cylinder are equivalent, this difference presents a somewhat worrying lack of consistence within all current FMT versions based on an approximate $\phi_{3}$ including at least rank-two tensors [31].

\section{Summary and discussion}

We discussed recent advances in fundamental measure theory (FMT) for non-spherical hard particles [51], which we summarize in table 1. Using fundamental mixed measure theory (FMMT) featuring a two-particle mixed weighted density, we were able to obtain a reliable liquid crystal phase diagram for rods. An important improvement has also been to build FMMT on the functional for hard spheres from [12] rather than that from [8] that was used almost exclusively in previous FMT studies. We also showed some analytical formulas that can be obtained by mapping a simpler phenomenological theory on density functional theory (DFT) for long-wave-length deformations.

We have focused on spherocylinders in this and past works as there is a wealth of simulation results with which we can compare our results. Other density functional theories are available for spherocylinders and some of these theories are as accurate as FMMT. Nevertheless, the strength of F(M)MT lies its formulation as a theory for mixtures of arbitrary convex (or not too non-convex) particles. Results that will be published elsewhere show that $\mathrm{F}(\mathrm{M}) \mathrm{MT}$ is as accurate for polyhedral rods as for spherocylinders [34]. In addition, the FMT results for the less demanding problem of hard-body fluids near a hard wall are very accurate for all non-spherical particles studied thus far $[6,28,30]$ (with the exception of tetrahedra [28]) indicating that F(M)MT is an accurate generic DFT for non-spherical hard particles.

We also showed that it is possible to obtain accurate results by truncating the expansion of the mixed weighted density in terms of products of weighted densities to second order and rescaling by a factor $\zeta(S)$ that depends on the local nematic order $S$. This calculation is much less computationally involved than the full FMMT calculation (even if we use a convergent expansion). Additionally, we considered the prefactor of the third term $\phi_{3}$ in the functional of the form given by equation (3), which is fixed in the original theory by the requirements that the third virial coefficient for hard spheres is exact and that the functional for the excess free energy depends only on the weighted densities. If we allow a different prefactor for highly anisotropic particles for which the former requirement arguably is less useful, we can improve the agreement between FMMT and simulations further.

We did not consider the high-density limit in this work. Groh and Mulder [57] studied the high-density limit for the hard-sphere crystal in DFT. All functionals considered by Groh and Mulder [57] predict the same logarithmic divergence of the free energy in the close-packing limit as cell theory [58, 59]. It is is not very difficult to show that any FMT that has an excess free energy functional that is bounded from below by a constant and that leads to a finite excess free energy for a single cavity in the zero-dimensional limit has this same divergence in the high-density limit, see the appendix. The next term in the free energy, which remains constant in the high-density limit, determines the accuracy of the theory. The predictions for this constant term from the versions of FMT that were derived from the zero-dimensional limit with $\mathrm{mul}$ tiple cavities $[8,12]$ agree well with computer simulations [57] for the high-density crystal of hard spheres. As FMMT is also correct for two cavities, we expect it also yields accurate predictions for the crystal of non-spherical particles. It should be noted that an expansion of FMMT is likely to converge slowly for the highly peaked density profile of a crystal.

Both the $\zeta(S)$ correction and the rescaling of $\phi_{3}$ complicate the implementation of FMT for mixtures of differently shaped particles, as a prefactor that depends on the particle 
shape is introduced in the theory. For the $\zeta(S)$ correction, this is not a problem if only one component is non-spherical: the rescaling is not required for spheres, whose treatment is exact at this order. For all other cases and also the third term $\phi_{3}$, the prefactor could be absorbed into new weighted densities which contain sums over the one-component weighted densities rescaled by a prefactor that depends on the species (and, possibly, on $S$ ) and that is equal to the optimal value for the one-component problem. In this case, one of the defining assumptions of FMT, that the excess free energy only depends on the density profile through the weighted densities, would be restored.

In discussing the rescaling of $\phi_{3}$, we have not specified how to determine the prefactor in general. We only showed that an additional prefactor smaller than one (we used 4/9) gives an improved theory for spherocylinders. We emphasize that this is not the prefactor that would give the exact third virial coefficient in the isotropic phase of spherocylinders; in fact, that prefactor would be much larger than one for long spherocylinders, which would not lead to an accurate theory for intermediate and large packing fractions.

We speculate that an improvement will be found generally when rescaling $\phi_{3}$ such that the FMMT equation of state (EOS) for the nematic phase in the limit of perfect alignment reduces to the EOS from a parallel particle FMT, i.e. an FMT specifically derived for perfectly aligned particles. Note that while deriving such a parallel particle FMT is possible [30, appendix B] [35] for arbitrary (convex) shapes (by using the zero-dimensional limit), the functional is not nearly as easily evaluated as the elegant functionals for perfectly aligned cubes [60] and cylinders [52] (this connects to the hitherto unsolved problem of finding closed form expressions for the mixed Minkowski volumes in integral geometry [39]).

Alternatively, one could make the prefactor of $\phi_{3}$ depend on the nematic order parameter $S$ and some fit parameters, which would be similar to the $\zeta(S)$ modification of $\phi_{2}$ [6] (see also section 3.1). The fit parameters can be obtained by considering the nematic phase with a prescribed orientational distribution $f_{S}(\hat{\mathbf{u}})$ that depends on the nematic order parameter $S$. The resulting FMT free energy could then be expanded with respect to $\rho_{0}$ and the coefficient of the third order could be fitted to the exact third virial coefficient for the orientational distribution $f_{S}(\hat{\mathbf{u}})$. An interesting topic for future work would be to determine the accuracy of this new functional for spherocylinders and other shapes, but it would require a numerical calculation of the virial coefficient for each $S$ value and particle shape of interest.

These implementation details aside, FMMT promises to be an accurate theory for the collective equilibrium behavior of non-spherical particles. As will certainly be discussed elsewhere in this special issue, DFT can be extended to nonequilibrium systems. All formulations thus far require an accurate free energy functional for the corresponding equilibrium system. Moreover, it has been demonstrated that an effective equilibrium theory can be used to describe active systems [61]. Therefore, our work is also important for non-equilibrium problems, such as colloidal swimmers or swimming bacteria that are non-spherical [62] in general.
Future work could also address phases with less continuous symmetries, such as a nematic phase near a hard wall. DFT studies of phases with no or only one continuous symmetry, such as the crystal of non-spherical particles or a columnar phase of disk-like particles, are also possible but highly computationally intensive, even when using (rescaled) tensorial weighted densities with a rank at most two instead of (a high-order expansion of) the full mixed weighted density. A problem of the same dimensionality as the crystal of uniaxial hard particles, the solvation of molecules in solvents consisting of linear molecules, has been studied using a DFT with a fully resolved density profile [63], which shows that problems with few continuous symmetries are also amendable to density functional treatments.

\section{Acknowledgments}

Financial support by the Deutsche Forschungsgemeinschaft (DFG) through the Cluster of Excellence 'Engineering of Advanced Materials' (EAM) in Erlangen is gratefully acknowledged.

\section{Appendix. High-density limit}

For all anisotropic hard-particle systems that we know of, the state in the high-density limit (in the bulk) is a crystal with an equilibrium density profile that is a sum of infinitely sharp peaks around the equilibrium one-particle configurations (position and orientation; we will restrict ourselves to onecomponent systems for simplicity). In fact, the width of the peak in each degree of freedom scales as $\alpha=\left(\rho / \rho_{\mathrm{cp}}\right)^{1 / 3}-1$, where $\rho_{\mathrm{cp}}$ is the (average) density at close packing. As a result, the ideal-gas free energy diverges as $-d_{f} \log (\alpha)$ in the limit $\alpha \rightarrow 0$, where $d_{f}$ is the number of degrees of freedom.

We make the assumptions that the excess free energy $\mathcal{F}_{\text {ex }}$ of a given FMT functional is bounded by a constant $-C_{0}$ from below and that $\mathcal{F}_{\text {ex }}$ remains finite for a single cavity in the zero-dimensional limit (the limit in which the cavity shrinks such that only one configuration remains accessible to the particle at a fixed average number of particles $\bar{N} \leqslant 1[11,12])$. In that limit, the ideal gas will show the same logarithmic divergence as in the high-density limit for the bulk. Therefore, the total single-cavity free energy has the same logarithmic divergence as exhibited by cell theory $[58,59]$.

Now consider again the crystal and define a cavity for every lattice site that contains the corresponding particle at its equilibrium configuration such that no two particles in different cavities may overlap (an explicit example of such a construction would be a (set) Voronoi tesselation). In the high-density limit, this means that whenever any two particles are in the same cavity they must overlap. This is, of course, the construction used in cell theory. The equilibrium density profile for an external potential that is the result of these cavities is a sum of sharp peaks that do not overlap. In addition, each weighted density is a sum of similarly non-overlapping functions as can be seen from the definition in equation (4) and the fact that the particles in their entirety (i.e. not just their 
centers) are confined inside to the cavities. Thus the intrinsic free energy separates into a sum of single-cavity free energies, such that it diverges in the same way as the cell theory free energy in the high-density limit, $\mathcal{F}=-d_{f} \log (\alpha)+\mathcal{O}(1)$ for $\alpha \rightarrow 0$.

We can connect this result to the bulk crystal by realizing that the intrinsic free energy functional evaluated at this density profile is larger than (or equal to [57]) its minimum (at fixed average density), which is the Helmholtz free energy of the bulk crystal, because the intrinsic free energy does not depend on the external potential. Thus, we have obtained an upper bound on the FMT free energy of the bulk crystal. The lower bound is much simpler as we have assumed $\mathcal{F}_{\text {ex }}>-C_{0}$; therefore, $\mathcal{F}>\mathcal{F}_{\text {id }}-C_{0}$. Since its lower and upper bound show the cell theory divergence, $-d_{f} \log (\alpha)+\mathcal{O}(1)$, the FMT free energy must also diverge in the same way.

\section{References}

[1] Sacanna S and Pine D J 2011 Curr. Opin. Colloid Interface 1696

[2] Allen M P, Evans G T, Frenkel D and Mulder B M 1993 Hard Convex Body Fluids (Advances in Chemical Physics vol 86) ed I Prigogine and S A Rice (Hoboken, NJ: Wiley) p 1-166

[3] Evans R 1979 Adv. Phys. 28143

[4] Tarazona P, Cuesta J and Martínez-Ratón Y 2008 Theory, Simulation of Hard-Sphere Fluids, Related Systems (Lecture Notes in Physics, vol 753) ed Á Mulero (Berlin: Springer) pp 247-341

[5] Hansen-Goos H and Mecke K 2009 Phys. Rev. Lett. 102018302

[6] Hansen-Goos H and Mecke K 2010 J. Phys.: Condens. Matter 22364107

[7] Löwen H 1994 Phys. Rep. 237249

[8] Tarazona P 2000 Phys. Rev. Lett. 84694

[9] Singh Y 1991 Phys. Rep. 207351

[10] Rosenfeld Y 1989 Phys. Rev. Lett. 63980

[11] Rosenfeld Y, Schmidt M, Löwen H and Tarazona P 1997 Phys. Rev. E 554245

[12] Tarazona P and Rosenfeld Y 1997 Phys. Rev. E 55 R4873

[13] Carnahan N F and Starling K E 1969 J. Chem. Phys. 51635

[14] Roth R, Evans R, Lang A and Kahl G 2002 J. Phys.: Condens. Matter 1412063

[15] Hansen-Goos H and Roth R 2006 J. Phys.: Condens. Matter 188413

[16] Oettel M, Görig S, Härtel A, Löwen H, Radu M and Schilling T 2010 Phys. Rev. E 82051404

[17] Somoza A M and Tarazona P 1990 Phys. Rev. A 41965

[18] Poniewierski A and Hołyst R 1988 Phys. Rev. Lett. 612461

[19] Graf H and Löwen H 1999 J. Phys.: Condens. Matter 111435

[20] Velasco E, Mederos L and Sullivan D E 2000 Phys. Rev. E 623708

[21] Bolhuis P and Frenkel D 1997 J. Chem. Phys. 106666

[22] Schmidt M 2001 Phys. Rev. E 63050201

[23] Esztermann A, Reich H and Schmidt M 2006 Phys. Rev. E 73011409

[24] Rosenfeld Y 1994 Phys. Rev. E 50 R3318
[25] Härtel A and Löwen H 2010 J. Phys.: Condens. Matter 22104112

[26] Härtel A, Blaak R and Löwen H 2010 Phys. Rev. E 81051703

[27] Fischermeier E, Marechal M and Mecke K 2014 J. Chem. Phys. 141194903

[28] Marechal M and Löwen H 2013 Phys. Rev. Lett. 110137801

[29] Marechal M, Zimmermann U and Löwen H 2012 J. Chem. Phys. 136144506

[30] Marechal M, Goetzke H H, Härtel A and Löwen H 2011 J. Chem. Phys. 135234510

[31] Wittmann R, Marechal M and Mecke K 2014 J. Chem. Phys. 141064103

[32] Wittmann R, Marechal M and Mecke K 2015 Europhys. Lett. 10926003

[33] Wittmann R and Mecke K 2014 J. Chem. Phys. 140104703

[34] Marechal M, Dussi S, Mecke K and Dijkstra M 2016 unpublished

[35] Marechal M, Korden S and Mecke K 2014 Phys. Rev. E 90042131

[36] Polson J M and Frenkel D 1997 Phys. Rev. E 56 R6260

[37] Poniewierski A and Sluckin T J 1991 Phys. Rev. A 436837

[38] Poniewierski A 1992 Phys. Rev. A 455605

[39] Schneider R and Weil W 2008 Stochastic and Integral Geometry (New York: Springer)

[40] Wittmann R, Marechal M and Mecke K 2015 Phys. Rev. E 91052501

[41] Press W H and Farrar G R 1990 Comput. Phys. 4190

[42] Galassi M, Davies J, Theiler J, Gough B, Jungman G, Alken P, Booth M and Rossi F 2009 GNU Scientific Library Reference Manual 3rd edn (Bristol: Network Theory)

[43] Wertheim M S 1994 Mol. Phys. 83519

[44] Gennes P D 1969 Phys. Lett. A 30454

[45] Gennes P G D 1971 Mol. Cryst. Liq. Cryst. 12193

[46] Virga E G 1995 Variational Theories for Liquid Crystals vol 8 (Boca Raton, FL: CRC Press)

[47] Fischermeier E, Bartuschat D, Preclik T, Marechal M and Mecke K 2014 Comput. Phys. Commun. 1853156

[48] Somoza A M and Tarazona P 1989 Phys. Rev. A 406069

[49] Poniewierski A and Hołyst R 1990 Phys. Rev. A 416871

[50] Hansen J-P and McDonald I R 1990 Theory of Simple Liquids 3rd edn (Amsterdam: Elsevier)

[51] Wittmann R 2015 Density functional theory for liquid crystals FAU Studies Mathematics \& Physics vol 5 (PhD thesis) (Erlangen: FAU University Press)

[52] Martínez-Ratón Y, Capitán J A and Cuesta J A 2008 Phys. Rev. E 77051205

[53] Reich H, Dijkstra M, van Roij R and Schmidt M 2007 J. Phys. Chem. B 1117825

[54] Frenkel D 1988 J. Phys. Chem. 925314

[55] Santos A 2012 Phys. Rev. E 86040102

[56] Hansen-Goos H, Mortazavifar M, Oettel M and Roth R 2015 Phys. Rev. E 91052121

[57] Groh B and Mulder B 2000 Phys. Rev. E 613811

[58] Kirkwood J G 1950 J. Chem. Phys. 18380

[59] Wood W W 1952 J. Chem. Phys. 201334

[60] Cuesta J A and Martínez-Ratón Y 1997 Phys. Rev. Lett. 783681

[61] Farage T F F, Krinninger P and Brader J M 2015 Phys. Rev. E 91042310

[62] Elgeti J, Winkler R G and Gompper G 2015 Rep. Prog. Phys. 78056601

[63] Borgis D, Gendre L and Ramirez R 2012 J. Phys. Chem. B 1162504 